Supporting Information for:

\title{
MAX-Phase Films Overcome Scaling Limitations to the Resistivity of Metal Thin Films
}

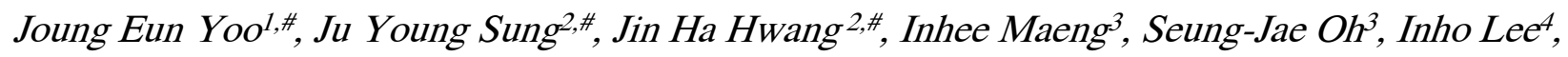
Ji Hoon Shim ${ }^{4,5}$, Sung Dug Kim¹, Du-Seop Yoon ${ }^{1}$, Seo Young Jang², Young Jae Kangl,*, and Sang Woon Lee?, *

${ }^{1}$ Material Research Center, SAIT, Samsung Electronics, Suwon, 16678, Republic of Korea

${ }^{2}$ Department of Energy Systems Research and Department of Physics, Ajou University, Suwon,

16499, Republic of Korea

${ }^{3}$ YUHS-KRIBB Medical Convergence Research Institute, Yonsei University, Seoul, 03722,

Republic of Korea

${ }^{4}$ Department of Chemistry, Pohang University of Science and Technology, Pohang, 37673,

Republic of Korea 
5Department of Physics, Pohang University of Science and Technology, Pohang, 37673,

Republic of Korea

Email: slee01@ajou.ac.kr, yjae.kang@samsung.com

This PDF file includes:

Supplementary Table S1, Figures S1-S2 


\begin{tabular}{|c|c|}
\hline V (at.\%) & Al (at.\%) \\
\hline 66.9 & 33.1 \\
\hline
\end{tabular}

Table S1. Stoichiometric ratio of $\mathrm{V}$ :Al in a 35-nm-thick $\mathrm{V}_{2} \mathrm{AlC}$ film analyzed using ICP-AES. 


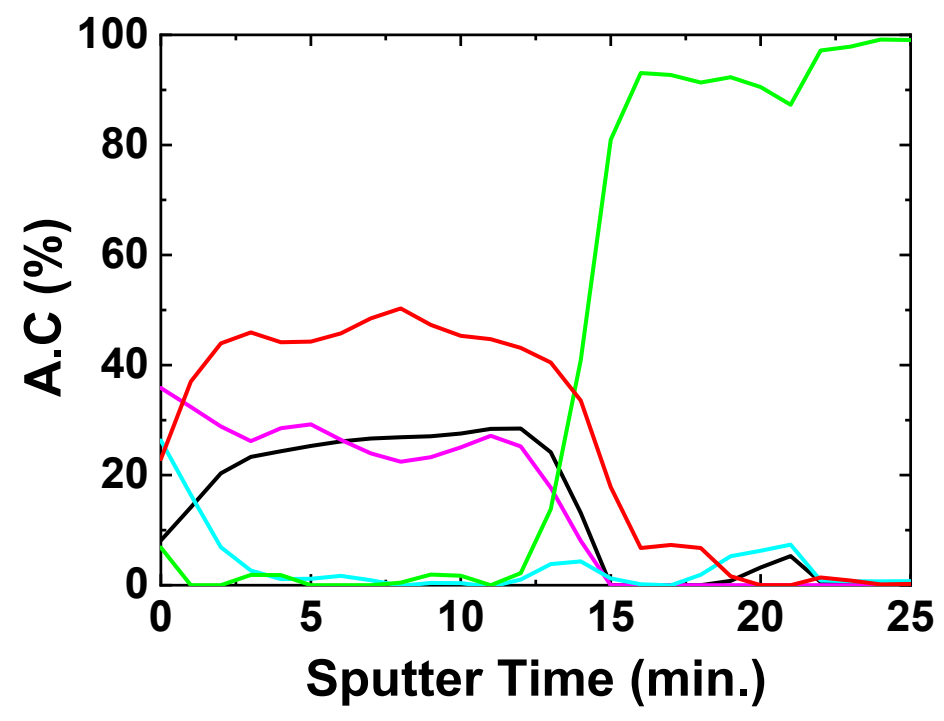

\begin{tabular}{|c|c|c|c|}
\hline $\mathrm{V}$ & $\mathrm{Al}$ & $\mathrm{O}$ & $\mathrm{C}$ \\
\hline 48.47 & 23.96 & 0.91 & 26.66 \\
& & & \\
\hline
\end{tabular}

Figure S1. XPS depth profiling spectra for a $35-\mathrm{nm}$-thick $\mathrm{V}_{2} \mathrm{AlC}$ film. The sputter rate was 2.5 $\mathrm{nm} \cdot \mathrm{min}^{-1}$ and the oxygen concentration was less than 1 at.\%. 
(a)

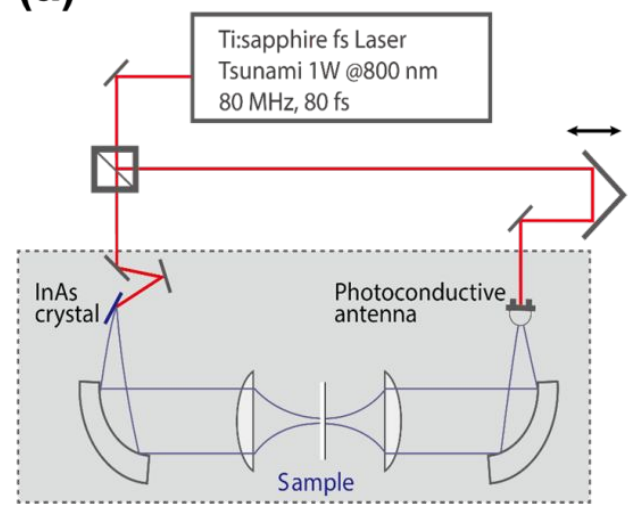

(b)

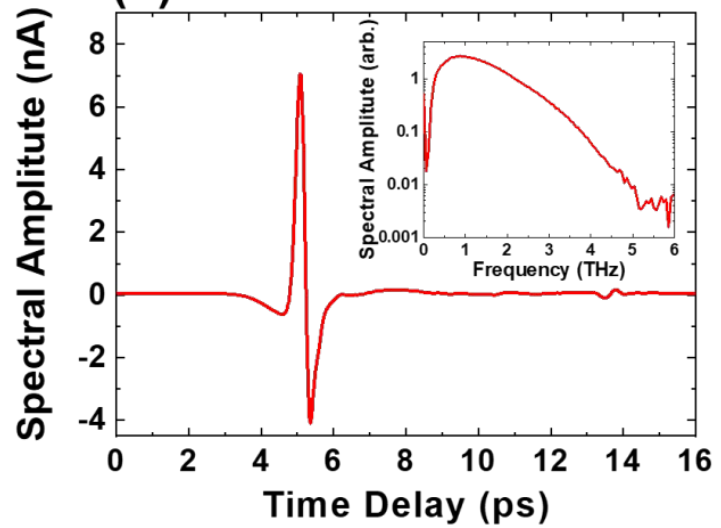

Figure S2. (a) Schematic of the experimental setup for THz-time domain spectroscopy (THzTDS). (b) TDS signal for a 35-nm-thick $\mathrm{V}_{2} \mathrm{AlC}$ film. Inset: spectrum corresponding to (b) after FTT. 